\title{
19. DIATOMS IN DSDP LEG 41 SITES
}

Hans-Joachim Schrader, ' Geologisch-Paläontologisches Institut und Museum der Universität Kiel, Olshausenstr. 40/60, D-2300 Kiel, F.R. Germany

\begin{abstract}
Marine planktonic diatom frustules are found with varying abundance and preservation in Cenozoic sediments of Sites 366 and 369. Displaced fresh-water diatoms and phytoliths are observed at Site 366 in Core 2, Hole 366A in Cores 1 and 2, and at Hole 369A with rare abundance in Cores 1 through 30 . No biostratigraphic investigation nor detailed taxonomy were made.
\end{abstract}

\section{INTRODUCTION}

Leg 41 of the Deep Sea Drilling Project, from Abidjan to Malaga drilled five sites, Sites 366 through 370 , on the eastern north Atlantic margin (Figure 1). Light microscope techniques were used exclusively to study opal phytoplankton, fresh-water diatoms, and phytoliths. Sample preparation followed the method of Schrader and Fenner (1976) for semiquantitative study. Diatom zonation partly used here is that of Schrader and Burckle (in press). No detailed biostratigraphic investigation was made for the Paleogene interval at Sites 366 and 369 because most of the assemblages are poorly preserved. No detailed taxonomic investigation was done on Leg 41 samples so some species were grouped and listed in Tables 1 and 2 as spp. All other species are described in Schrader (1974), Kolbe (1954), Burckle (1972), Hustedt (1930-1959), and Simonsen (1974).

Samples barren in diatoms are marked on the summary figures with an arrow and are tabulated.

The aim of this investigation is to present information of the diatom content in order to facilitate subsequent detailed diatom studies on Leg 41 sediments, especially for the Oligocene to Eocene interval.

\section{RESULTS}

\section{Site 366}

This continuously cored sedimentary sequence ranges in age from Holocene to Maestrichtian and contains marine planktic diatoms. Their abundance ranges from trace to abundant and their preservation varies from poor to good in the Pleistocene and early Miocene through late Eocene interval. Detailed information is shown in Figure 2. Samples studied but barren are listed in Figure 2 (arrows).

Displaced fresh-water diatoms and phytoliths are common in the Pleistocene and rare in the early Miocene (Figure 2, triangles).

Selected ranges of important species are documented in Figure 3.

\footnotetext{
'Present address: School of Oceanography, Oregon State University, Corvallis, Oregon.
}

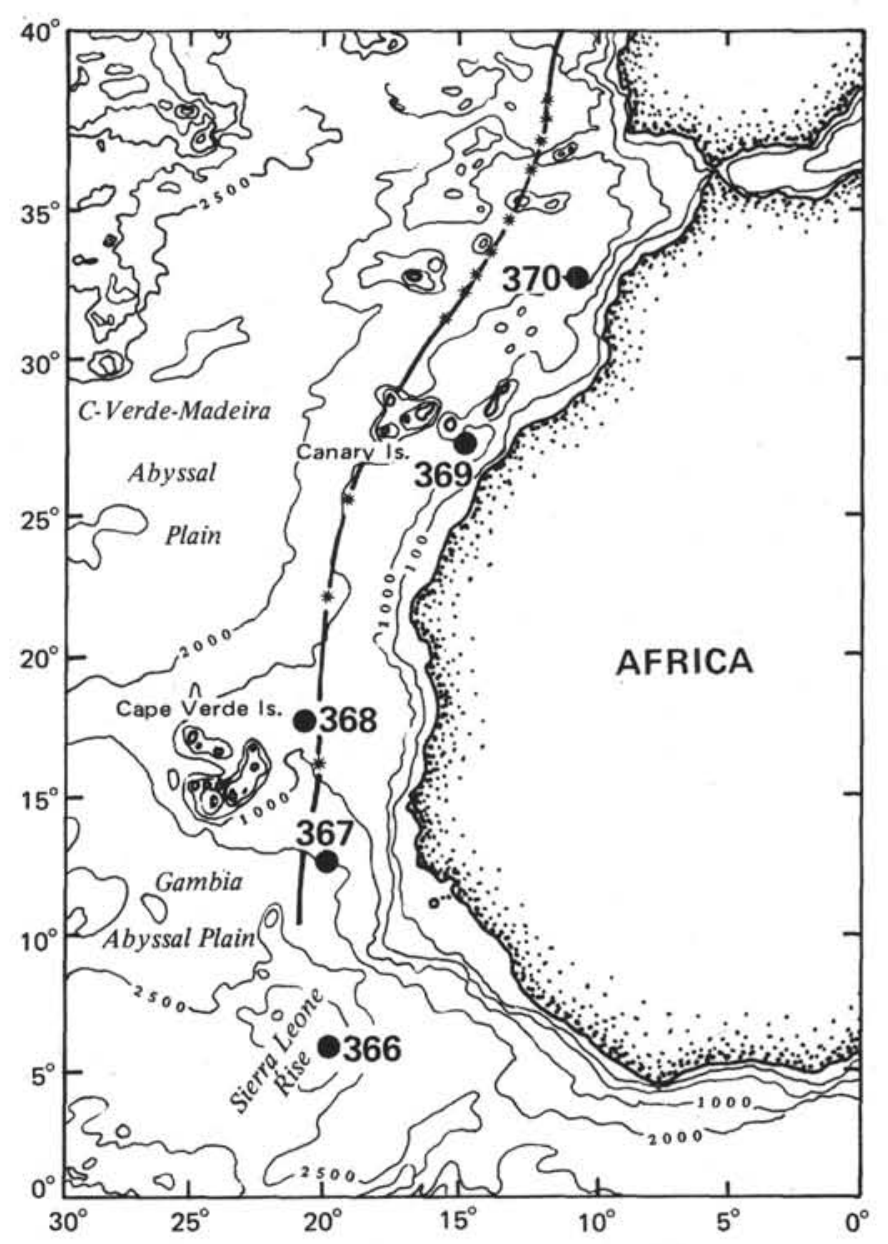

Figure 1. Localities of DSDP Leg 41 drill sites.

\section{Site 367}

All 126 samples from Cores 1-37 (except Cores 7, 8, and 11) obtained from the Cape Verde Basin site were barren in opal phytoplankton remains (Figure 4).

\section{Site 368}

All 244 samples from Cores 2-63 (except Cores 48, 60 , and 61 ) of the mostly terrigenous sediment section at the Cape Verde Rise site were barren in opal phytoplankton remains. 


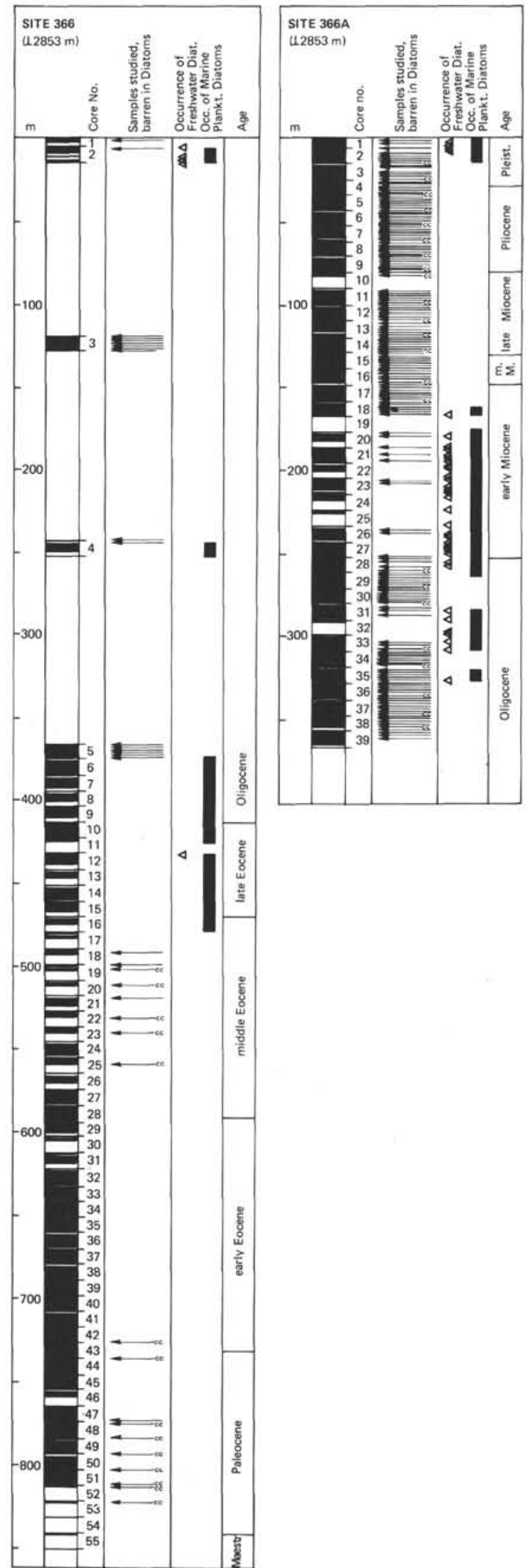

Figure 2. Site 366 summary figure. Ages are taken from the site reports (this volume). Arrow indicate samples barren in opal phytoplankton. 


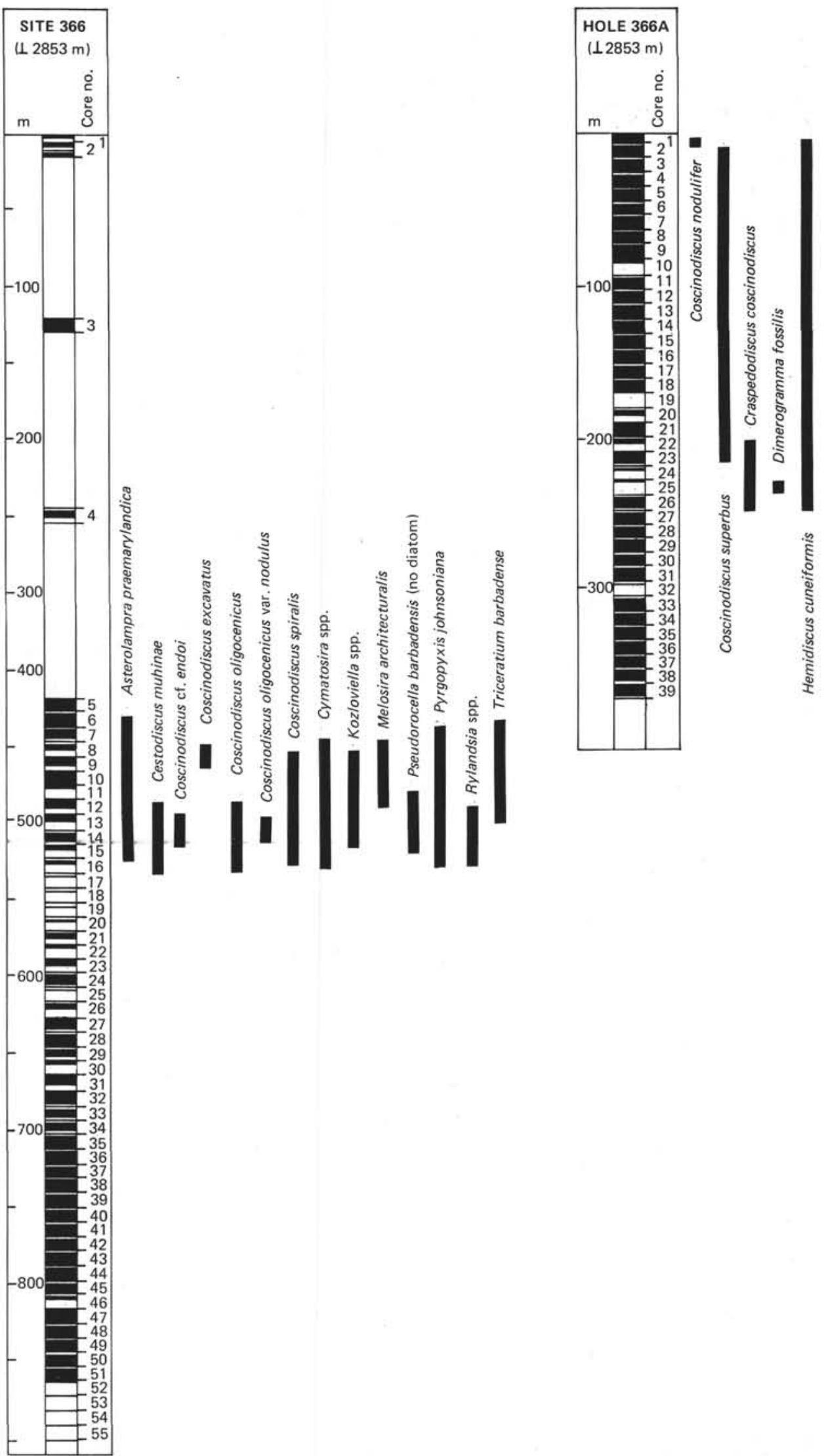

Figure 3. Occurrence of selected diatoms at Site 366 and Hole $366 \mathrm{~A}$ (range chart). 
TABLE 1

Holes 336/336A

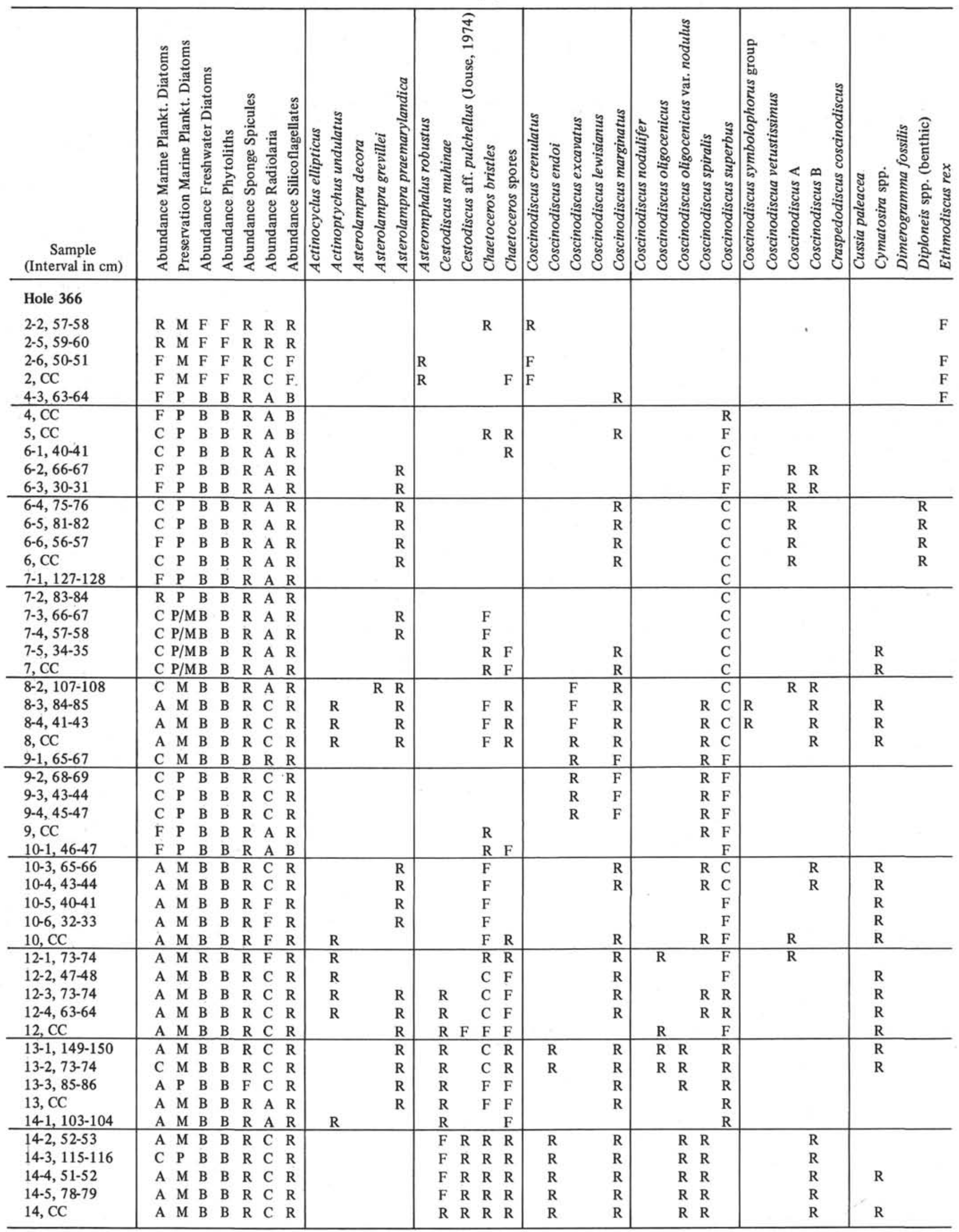


TABLE 1 - Continued

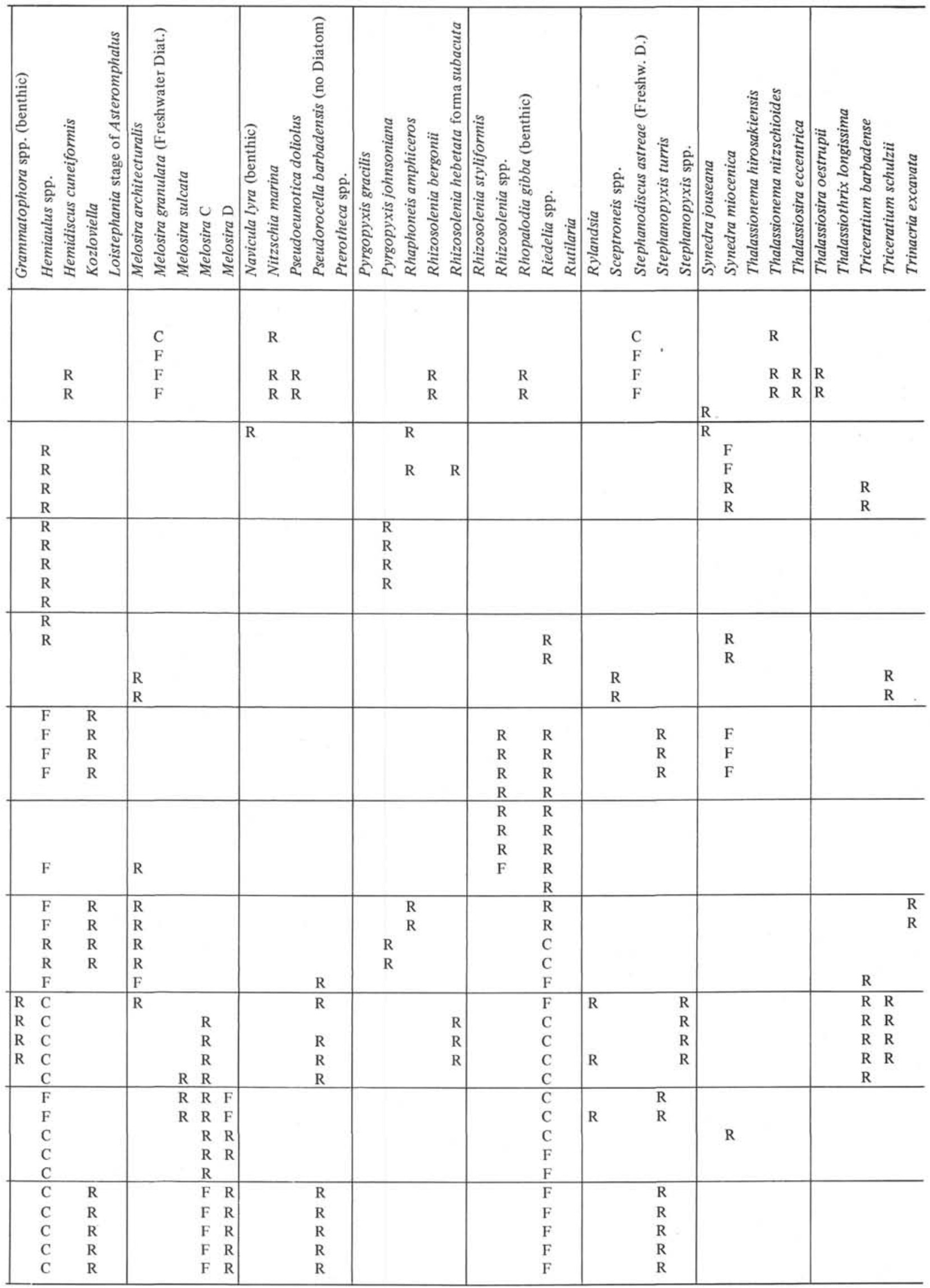


TABLE 1 - Continued

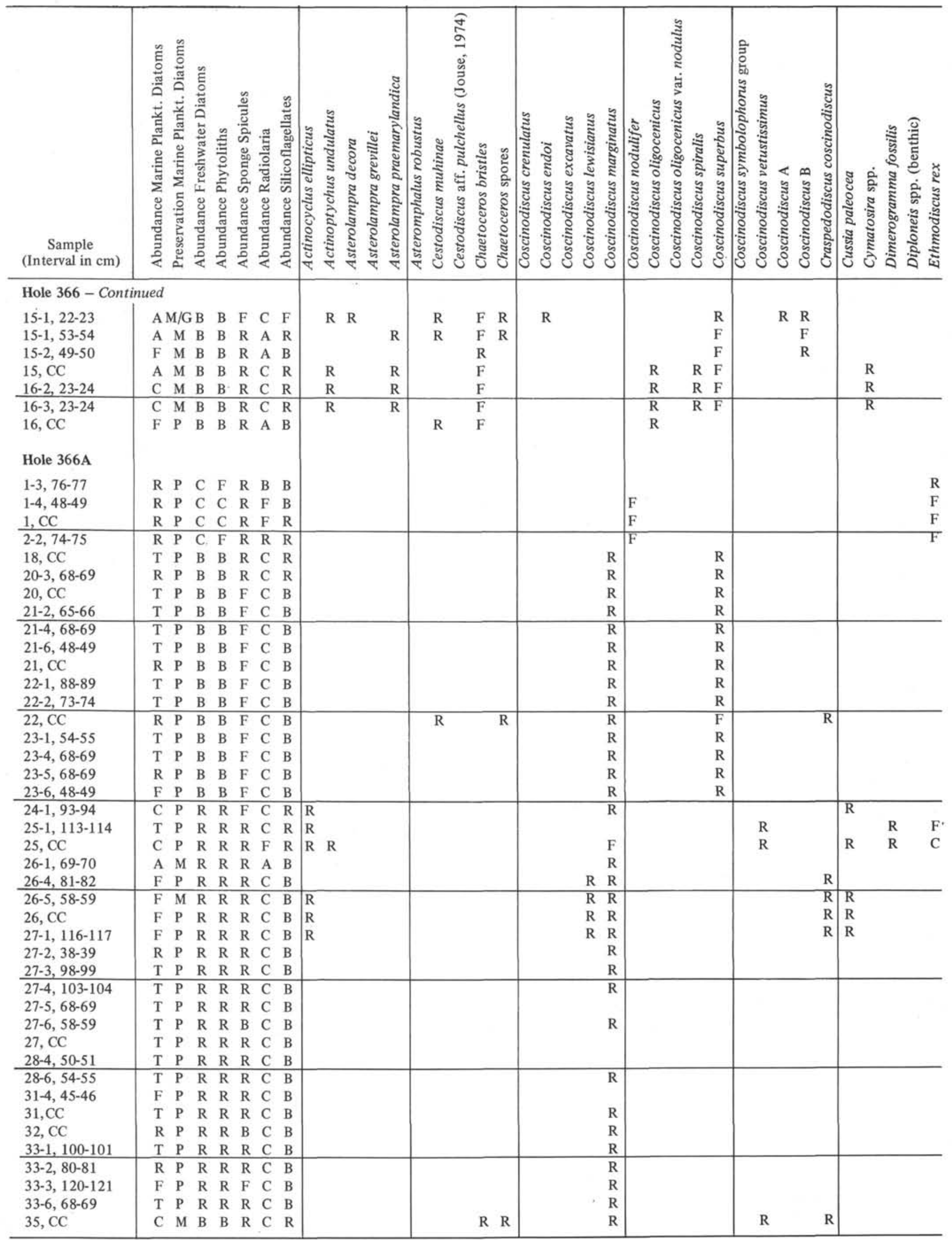


TABLE 1 - Continued

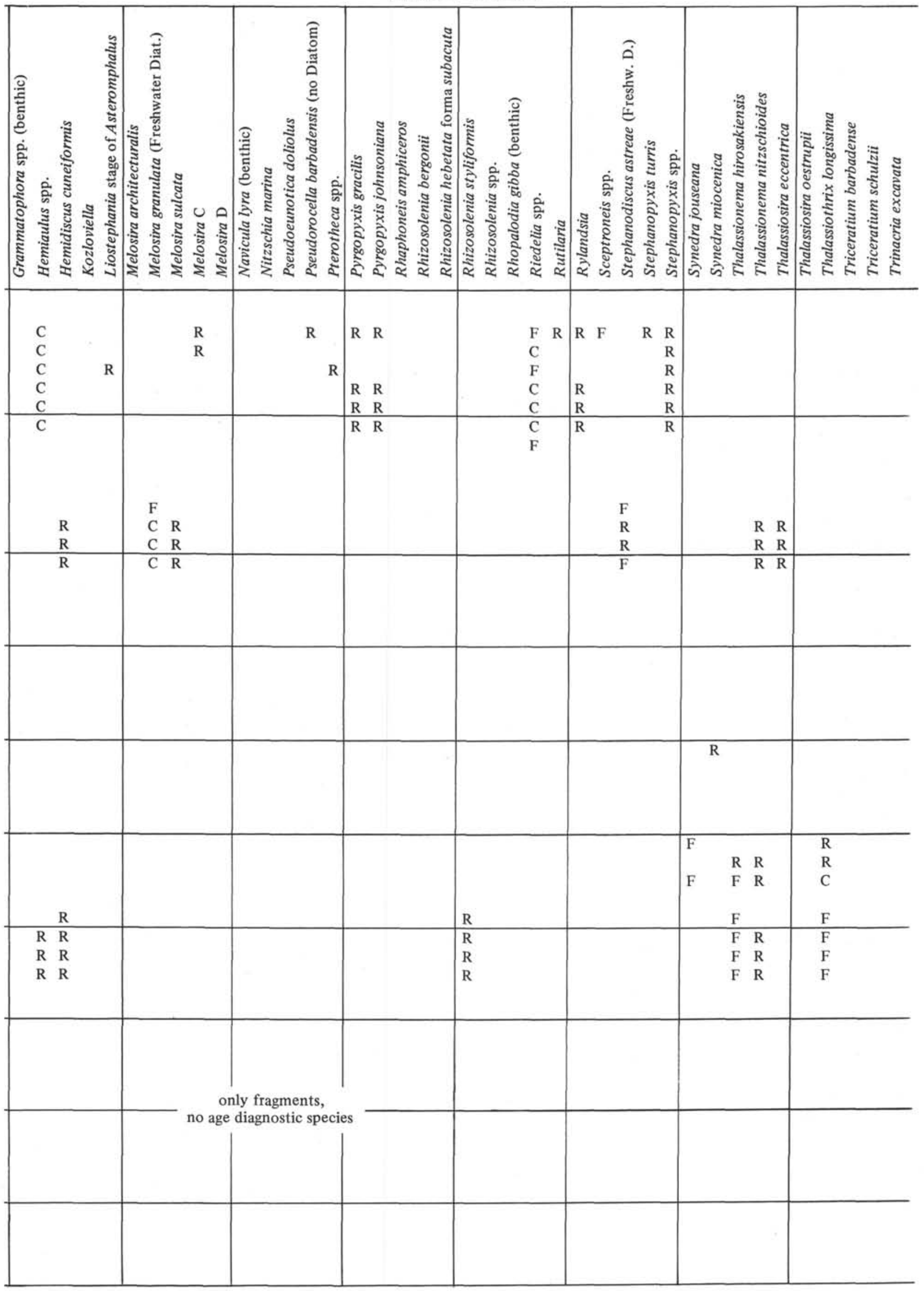


TABLE 2

Hole 369A

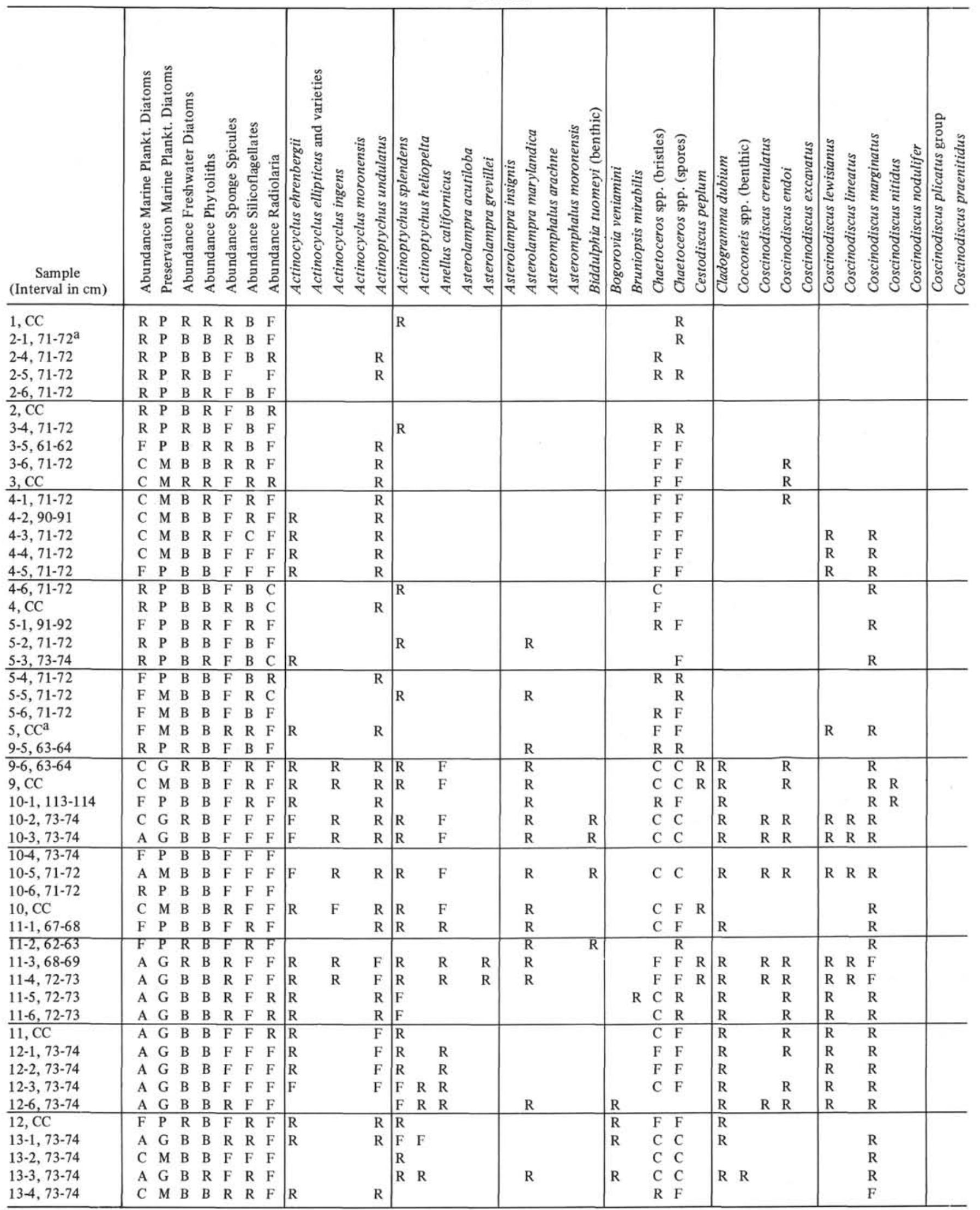


TABLE 2 - Continued

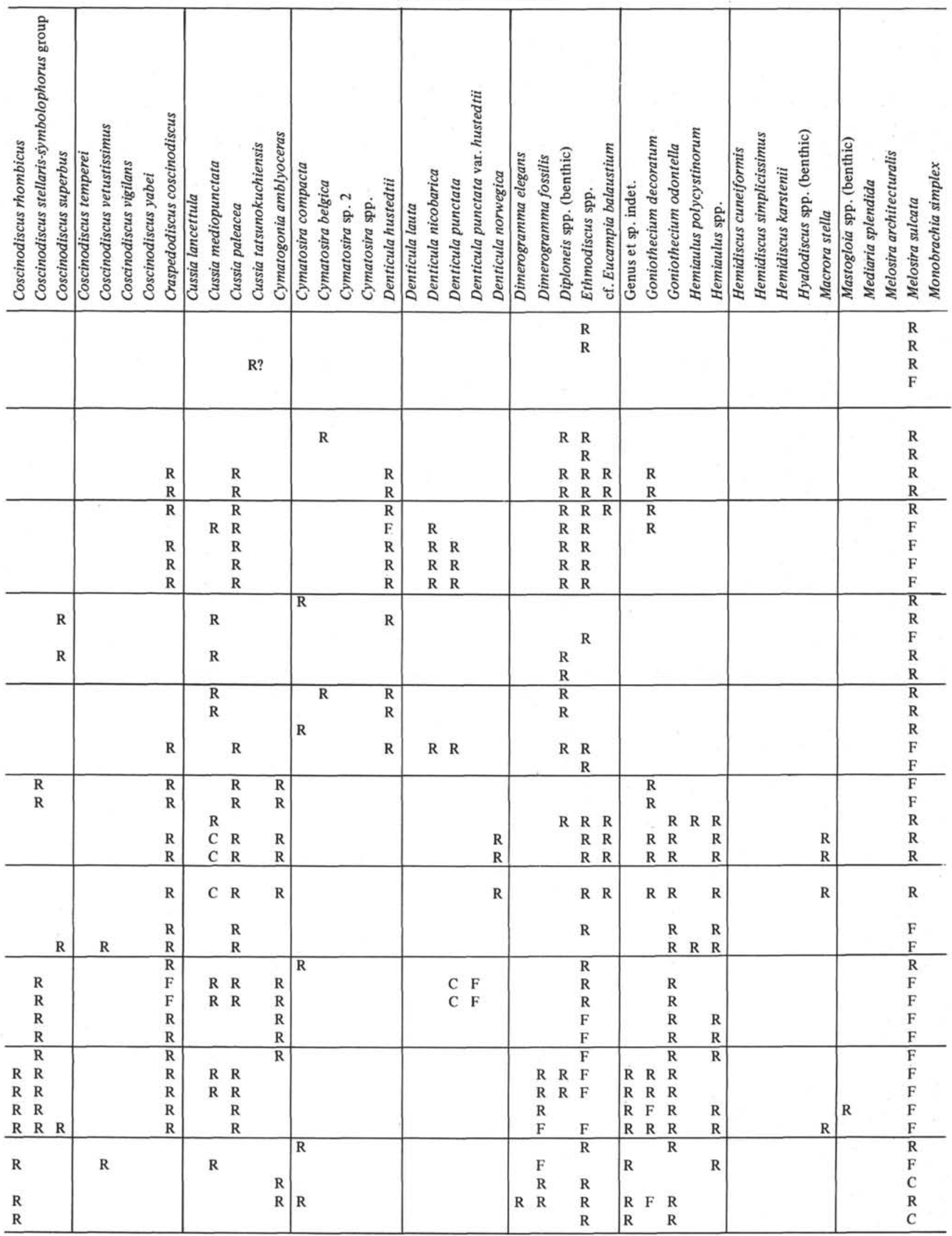


TABLE 2 - Continued

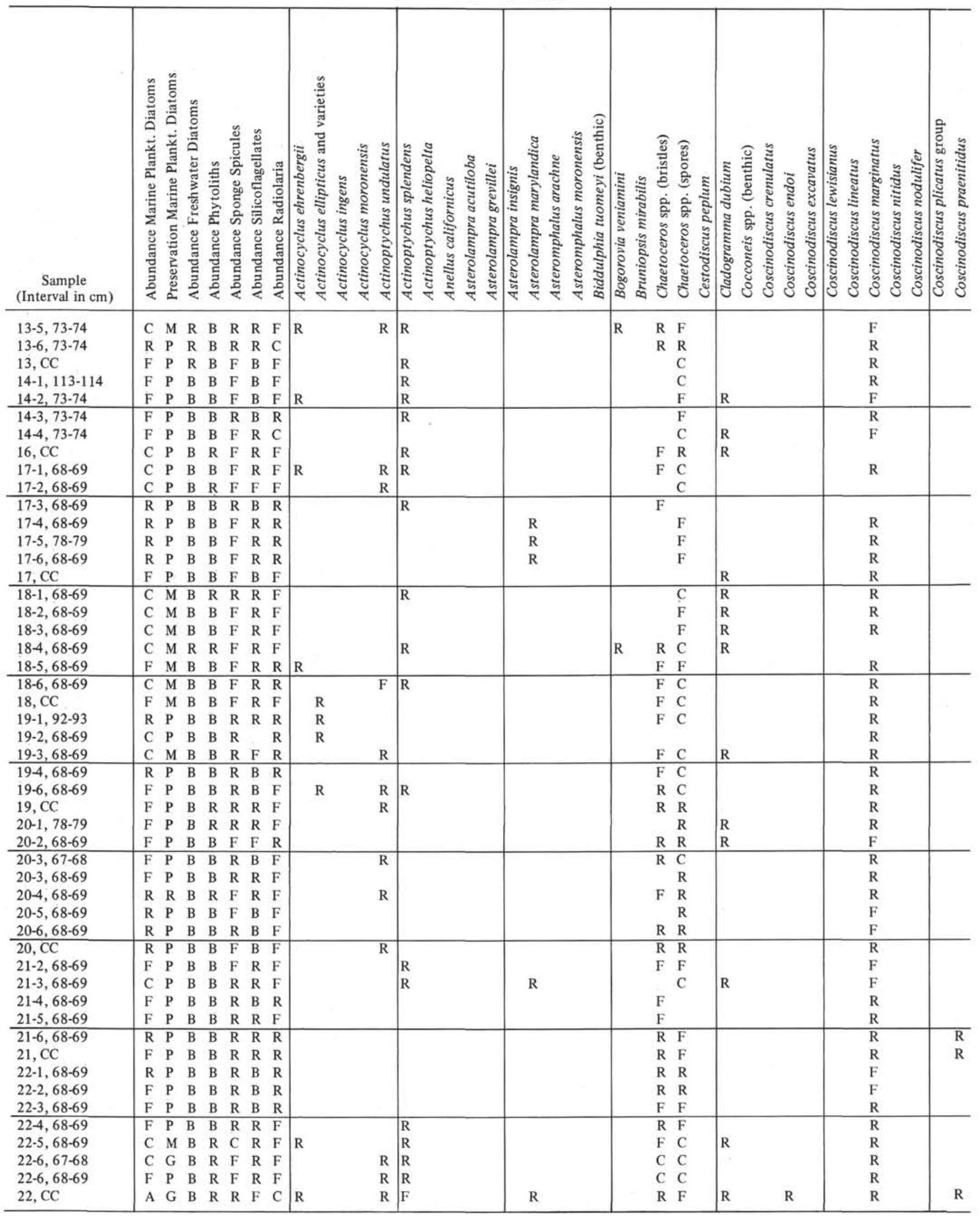


TABLE 2 - Continued

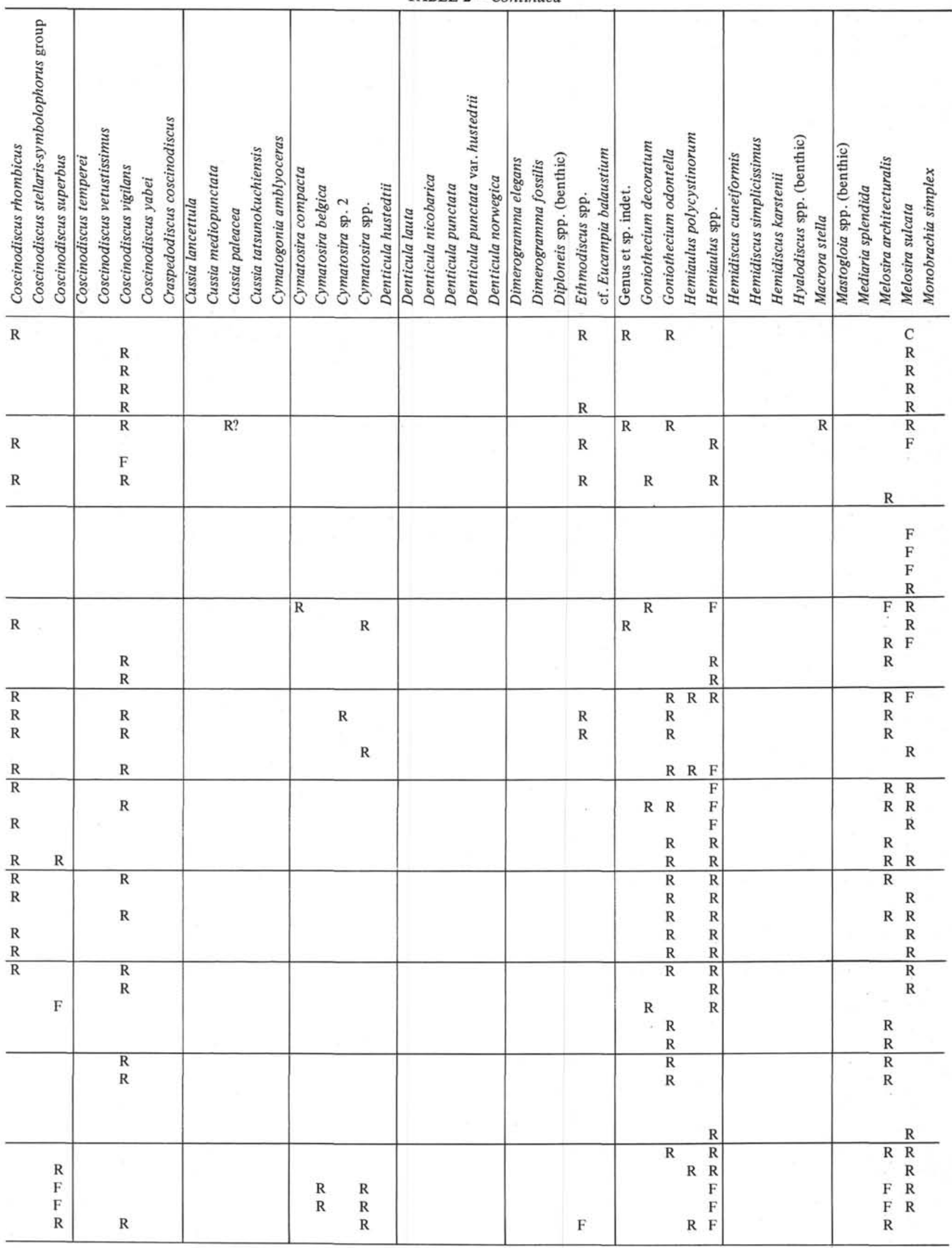


TABLE 2 - Continued

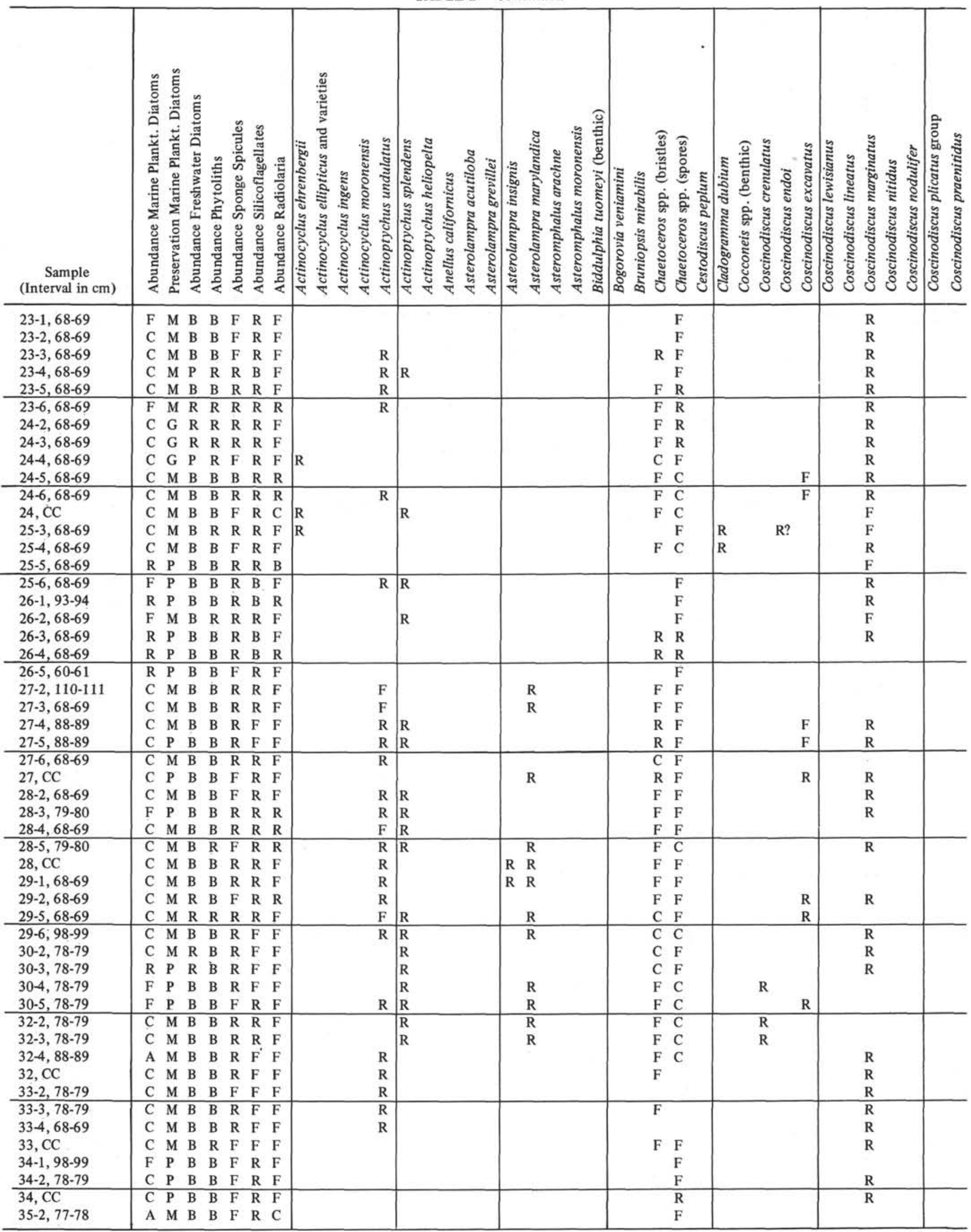

\footnotetext{
${ }^{\mathrm{a}}$ Ash
} 
TABLE 2 - Continued

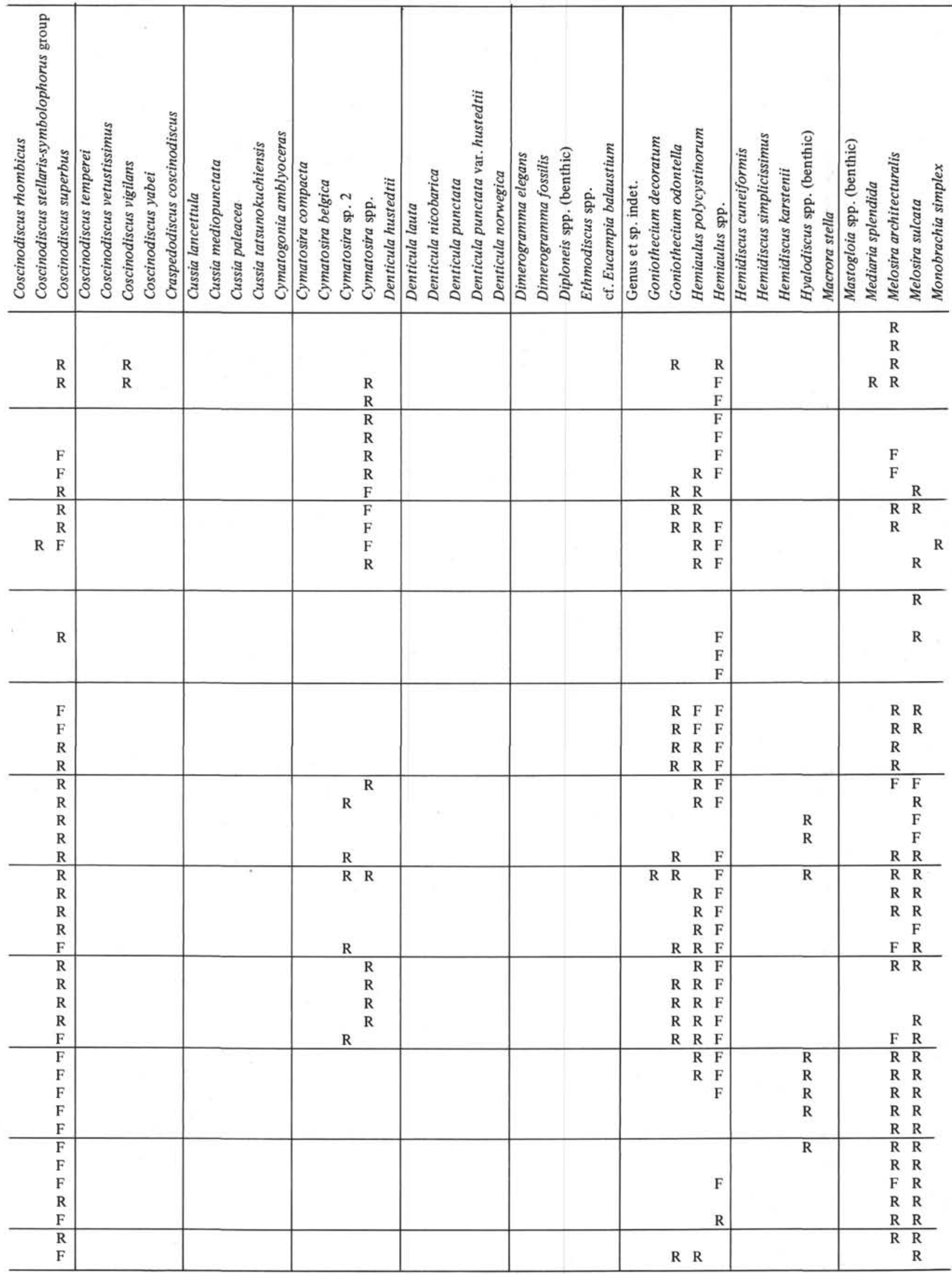


TABLE 2 - Continued

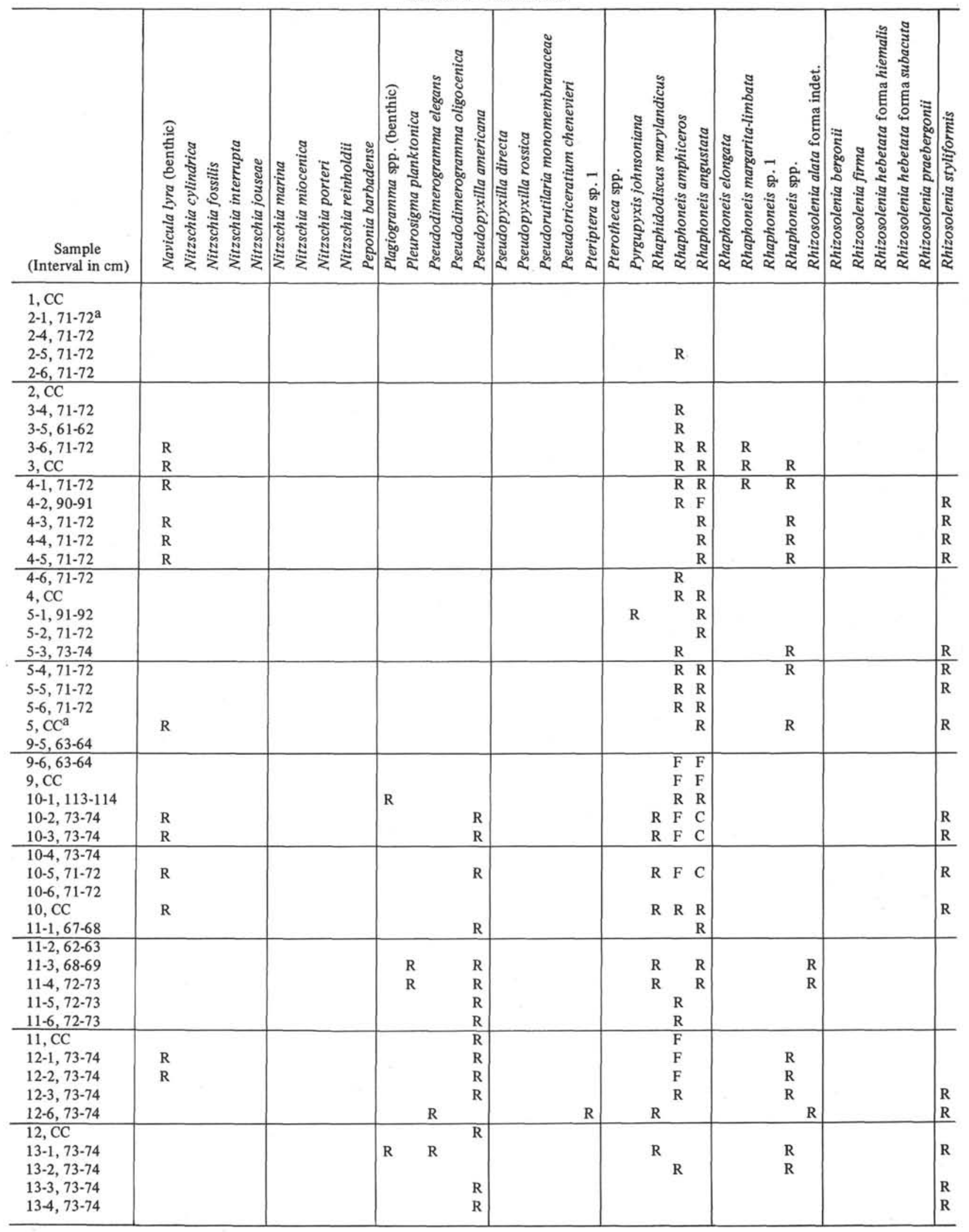




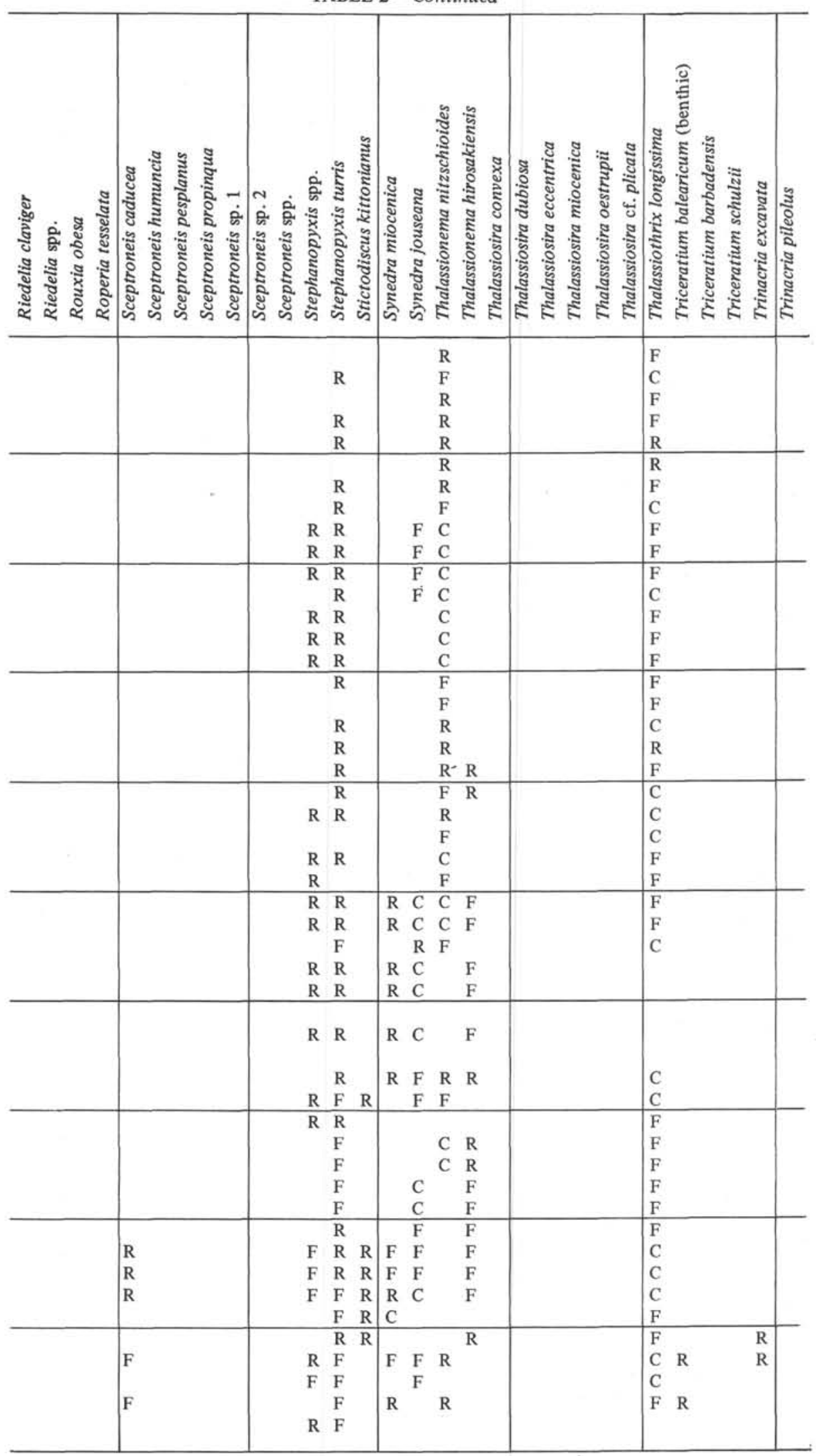


TABLE 2 - Continued

\begin{tabular}{|c|c|c|c|c|c|c|c|c|}
\hline $\begin{array}{c}\text { Sample } \\
\text { (Interval in } \mathrm{cm})\end{array}$ & 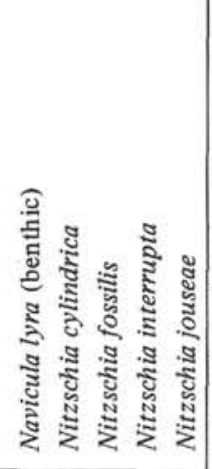 & 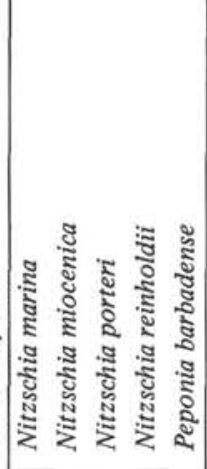 & 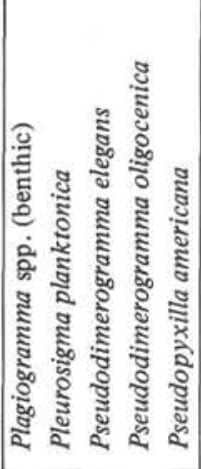 & 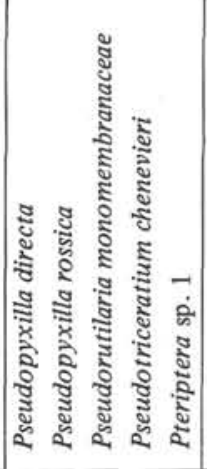 & 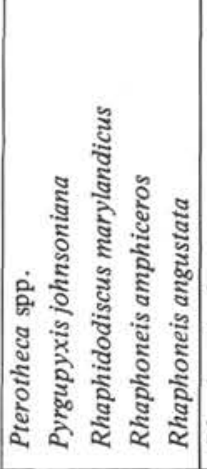 & 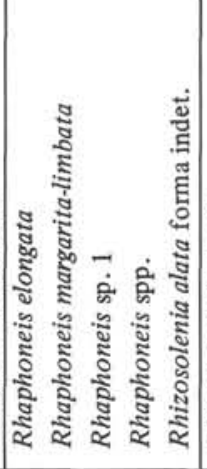 & 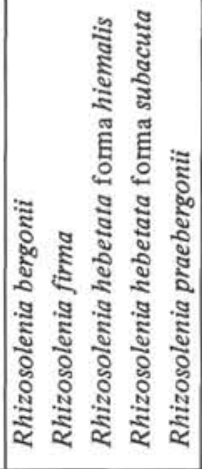 & 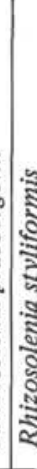 \\
\hline $\begin{array}{l}13-5,73-74 \\
13-6,73-74 \\
13, \mathrm{CC} \\
14-1,113-114 \\
14-2,73-74\end{array}$ & & & $\mathrm{R}$ & & $\begin{array}{l}\mathrm{R} \\
\mathrm{R}\end{array}$ & R & & R \\
\hline $\begin{array}{l}14-3,73-74 \\
14-4,73-74 \\
16, \mathrm{CC} \\
17-1,68-69 \\
17-2,68-69 \\
\end{array}$ & $\mathbf{R}$ & & $\begin{array}{l}\mathrm{R} \\
\mathrm{R} \\
\mathrm{R}\end{array}$ & & $\mathrm{R}$ & F & & $\begin{array}{l}\mathrm{R} \\
\mathrm{R}\end{array}$ \\
\hline $\begin{array}{l}17-3,68-69 \\
17-4,68-69 \\
17-5,78-79 \\
17-6,68-69 \\
17, \text { CC }\end{array}$ & & & & & & & & $\begin{array}{l}\mathrm{R} \\
\mathrm{R} \\
\mathrm{R}\end{array}$ \\
\hline $\begin{array}{l}18-1,68-69 \\
18-2,68-69 \\
18-3,68-69 \\
18-4,68-69 \\
18-5,68-69\end{array}$ & & $\mathrm{R}$ & $\mathrm{R}$ & & $\begin{array}{l}\mathrm{R} \\
\mathrm{R} \\
\mathrm{R}\end{array}$ & $\mathrm{R}$ & & $\mathrm{R}$ \\
\hline $\begin{array}{l}18-6,68-69 \\
18, C C \\
19-1,92-93 \\
19-2,68-69 \\
19-3,68-69\end{array}$ & $\mathrm{R}$ & & $\mathrm{F}$ & & $\begin{array}{l}\mathrm{R} \\
\mathrm{R} \\
\mathrm{R} \\
\mathrm{R} \\
\mathrm{R}\end{array}$ & $\begin{array}{ll}\mathrm{R} & \\
\mathrm{R} \\
\mathrm{R}\end{array}$ & $\mathrm{R}$ & $\mathrm{R}$ \\
\hline $\begin{array}{l}19-4,68-69 \\
19-6,68-69 \\
19, \text { CC } \\
20-1,78-79 \\
20-2,68-69\end{array}$ & & & $\begin{array}{l}\mathrm{R} \\
\mathrm{R}\end{array}$ & $\begin{array}{l}\mathrm{R} \\
\mathrm{R}\end{array}$ & R & R & & \\
\hline $\begin{array}{l}20-3,67-68 \\
20-3,68-69 \\
20-4,68-69 \\
20-5,68-69 \\
20-6,68-69\end{array}$ & $\mathrm{R}$ & J & & & & $\mathrm{R}$ & & \\
\hline $\begin{array}{l}20, \mathrm{CC} \\
21-2,68-69 \\
21-3,68-69 \\
21-4,68-69 \\
21-5,68-69\end{array}$ & & & & $\mathrm{R}$ & $\begin{array}{l}\mathrm{R} \\
\mathrm{R}\end{array}$ & $\mathrm{R}$ & & \\
\hline $\begin{array}{l}21-6,68-69 \\
21, C C \\
22-1,68-69 \\
22-2,68-69 \\
22-3,68-69\end{array}$ & & & & $\begin{array}{l}\mathrm{R} \\
\mathrm{R}\end{array}$ & $\mathrm{R}$ & $\begin{array}{l}\mathrm{R} \\
\mathrm{R}\end{array}$ & & \\
\hline $\begin{array}{l}22-4,68-69 \\
22-5,68-69 \\
22-6,67-68 \\
22-6,68-69 \\
22, \text { CC }\end{array}$ & $\begin{array}{l}\mathrm{R} \\
\mathrm{R} \\
\mathrm{R}\end{array}$ & & & $\begin{array}{l}\mathrm{R} \\
\mathrm{R}\end{array}$ & $\begin{array}{l}\mathrm{R} \\
\mathrm{R} \\
\mathrm{R}\end{array}$ & $\begin{array}{l}\mathrm{R} \\
\mathrm{F}\end{array}$ & $\begin{array}{l}\mathrm{R} \\
\mathrm{R}\end{array}$ & \\
\hline
\end{tabular}


TABLE 2 - Continued

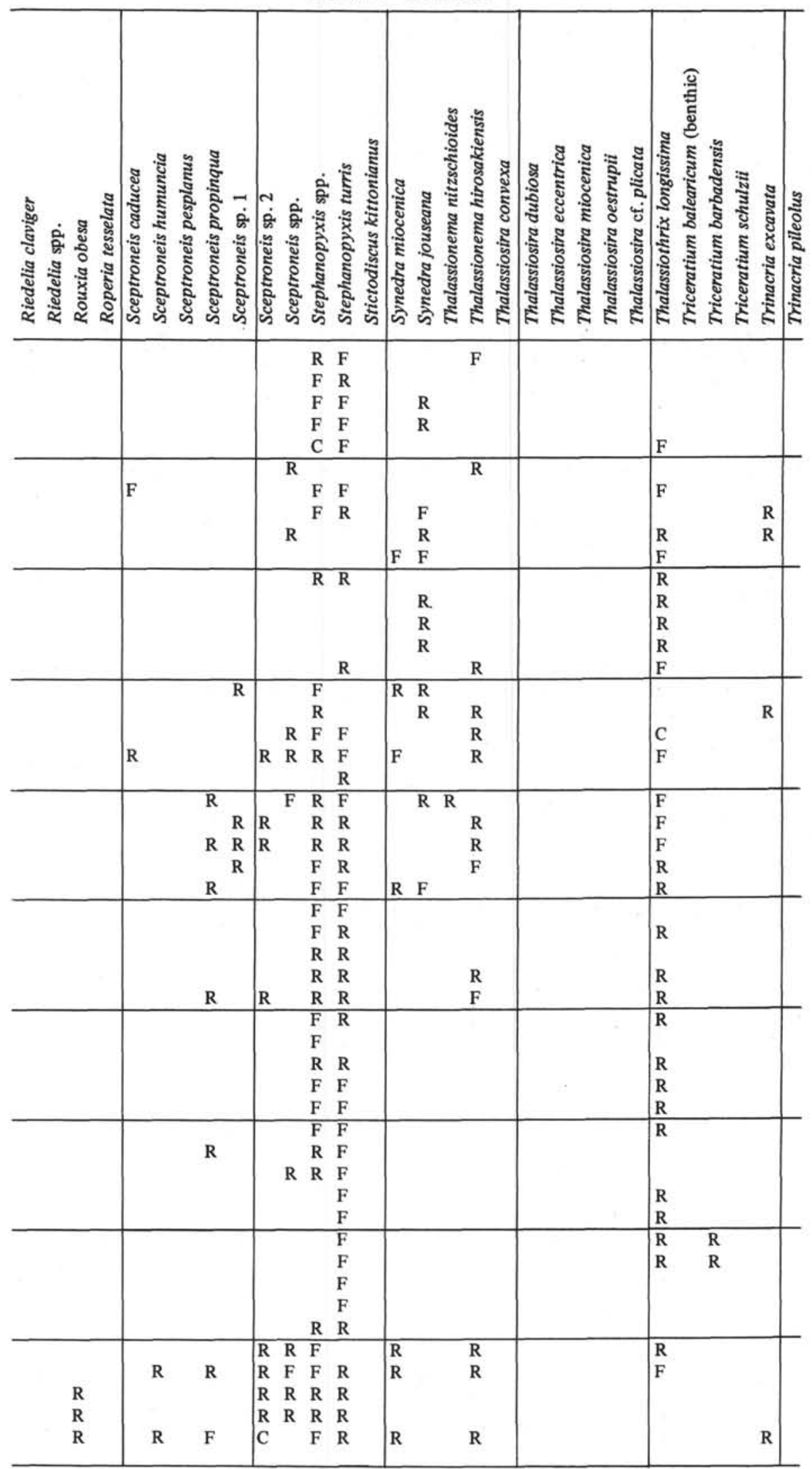


TABLE 2 - Continued

\begin{tabular}{|c|c|c|c|c|c|c|c|c|c|c|}
\hline $\begin{array}{c}\text { Sample } \\
\text { (Interval in } \mathrm{cm} \text { ) }\end{array}$ & 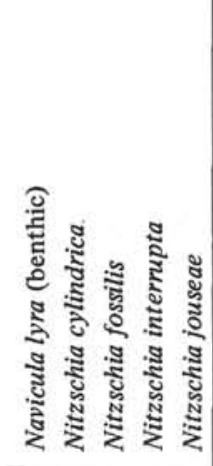 & 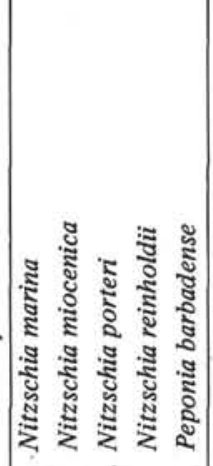 & 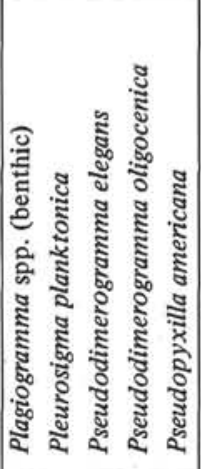 & 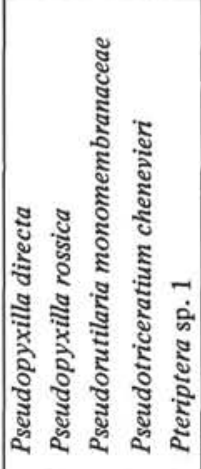 & 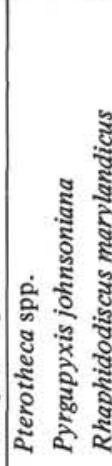 & 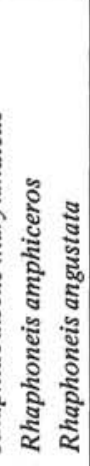 & 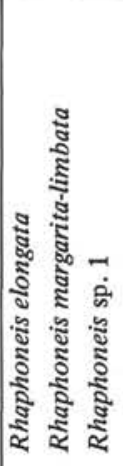 & 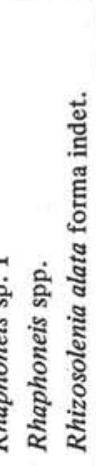 & 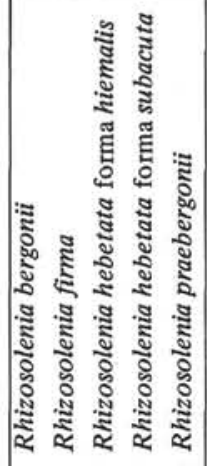 & 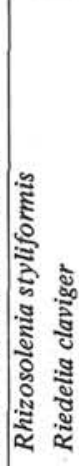 \\
\hline $\begin{array}{l}23-1,68-69 \\
23-2,68-69 \\
23-3,68-69 \\
23-4,68-69 \\
23-5,68-69\end{array}$ & $\begin{array}{l}\mathrm{R} \\
\mathrm{R}\end{array}$ & & $\mathrm{R}$ & $\begin{array}{lll} & & \text { R } \\
& & \text { R } \\
\mathrm{R} & \mathrm{R}\end{array}$ & $\begin{array}{l}\mathrm{R} \\
\mathrm{R} \\
\mathrm{R}\end{array}$ & $\begin{array}{l}\mathrm{R} \\
\mathrm{R} \\
\mathrm{R} \\
\mathrm{R}\end{array}$ & & $\begin{array}{l}\mathrm{R} \\
\mathrm{R}\end{array}$ & $\begin{array}{l}\mathrm{R} \\
\mathrm{R}\end{array}$ & $R$ \\
\hline $\begin{array}{l}23-6,68-69 \\
24-2,68-69 \\
24-3,68-69 \\
24-4,68-69 \\
24-5,68-69\end{array}$ & & & $\begin{array}{ll} & \mathrm{R} \\
\mathrm{R} \\
\mathrm{R} \\
\mathrm{R} & \end{array}$ & $\begin{array}{l}\mathrm{R} \\
\mathrm{R} \\
\mathrm{R} \\
\mathrm{R} \\
\mathrm{R}\end{array}$ & $\mathrm{R}$ & $\begin{array}{l}\mathrm{R} \\
\mathrm{F} \\
\mathrm{F} \\
\mathrm{R} \\
\mathrm{R}\end{array}$ & $\mathrm{R}$ & $\begin{array}{l}\mathrm{F} \\
\mathrm{F} \\
\mathrm{F} \\
\mathrm{R}\end{array}$ & $\mathbf{R}$ & R \\
\hline $\begin{array}{l}24-6,68-69 \\
24, C C \\
25-3,68-69 \\
25-4,68-69 \\
25-5,68-69 \\
\end{array}$ & & & \begin{tabular}{ll|}
$\mathrm{R}$ & \\
$\mathrm{R}$ & \\
& $\mathrm{R}$
\end{tabular} & $\mathrm{R}^{\mathrm{R}}$ & $\mathrm{R}$ & $\begin{array}{l}\mathrm{R} \\
\mathrm{R} \\
\mathrm{R} \\
\mathrm{R}\end{array}$ & \begin{tabular}{|lll}
$\mathrm{R}$ & & \\
& $\mathrm{R}$ \\
\end{tabular} & $\begin{array}{l}\mathrm{R} \\
\mathrm{R} \\
\mathrm{F}\end{array}$ & $\mathrm{R}$ & $\begin{array}{l}\mathrm{R} \\
\mathrm{R} \\
\mathrm{R}\end{array}$ \\
\hline $\begin{array}{l}25-6,68-69 \\
26-1,93-94 \\
26-2,68-69 \\
26-3,68-69 \\
26-4,68-69 \\
\end{array}$ & & & & & & $\mathrm{R}$ & & F & $\mathrm{R}$ & \\
\hline $\begin{array}{l}26-5,60-61 \\
27-2,110-111 \\
27-3,68-69 \\
27-4,88-89 \\
27-5,88-89 \\
\end{array}$ & & & $\begin{array}{ll}\mathrm{R} & \mathrm{R} \\
\mathrm{R} & \mathrm{R} \\
& \mathrm{R} \\
& \mathrm{R}\end{array}$ & & & $\begin{array}{l}\mathrm{F} \\
\mathrm{F}\end{array}$ & $\begin{array}{l}\mathrm{R} \\
\mathrm{R} \\
\mathrm{R} \\
\mathrm{R}\end{array}$ & 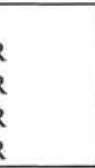 & $\begin{array}{l}R \\
R \\
R\end{array}$ & R \\
\hline $\begin{array}{l}27-6,68-69 \\
27, \mathrm{CC} \\
28-2,68-69 \\
28-3,79-80 \\
28-4,68-69 \\
\end{array}$ & 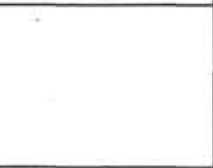 & & & & $\mathbf{R}$ & $\begin{array}{l}F \\
F\end{array}$ & $\begin{array}{l}\mathrm{R} \\
\mathrm{R} \\
\mathrm{R}\end{array}$ & $\begin{array}{l}\mathrm{R} \\
\mathrm{F} \\
\mathrm{F} \\
\mathrm{F}\end{array}$ & $\mathbf{R}$ & \\
\hline $\begin{array}{l}28-5,79-80 \\
28, C C \\
29-1,68-69 \\
29-2,68-69 \\
29-5,68-69 \\
\end{array}$ & & $\mathbf{R}$ & $\begin{array}{l}\mathrm{R} \\
\mathrm{R}\end{array}$ & $\begin{array}{l}\mathrm{R} \\
\mathrm{R}\end{array}$ & $\begin{array}{l}\mathrm{R} \\
\mathrm{F}\end{array}$ & & & $\begin{array}{l}\mathrm{R} \\
\mathrm{F}\end{array}$ & F & \begin{tabular}{|l} 
R \\
F \\
F \\
F
\end{tabular} \\
\hline $\begin{array}{l}29-6,98-99 \\
30-2,78-79 \\
30-3,78-79 \\
30-4,78-79 \\
30-5,78-79\end{array}$ & & & & R & $\begin{array}{l}\mathrm{R} \\
\mathrm{R} \\
\mathrm{R} \\
\mathrm{R} \\
\mathrm{F}\end{array}$ & $\begin{array}{l}\mathrm{R} \\
\mathrm{R} \\
\mathrm{R}\end{array}$ & $\mathrm{R}$ & & $\mathbf{R}$ & R \\
\hline $\begin{array}{l}32-2,78-79 \\
32-3,78-79 \\
32-4,88-89 \\
32, \text { CC } \\
33-2,78-79\end{array}$ & & & & & $\begin{array}{l}\mathrm{R} \\
\mathrm{R} \\
\mathrm{F}\end{array}$ & & & $\begin{array}{l}\mathrm{F} \\
\mathrm{R}\end{array}$ & $\begin{array}{l}\mathrm{R} \\
\mathrm{R} \\
\mathrm{R} \\
\mathrm{R} \\
\mathrm{R}\end{array}$ & \\
\hline $\begin{array}{l}33-3,78-79 \\
33-4,68-69 \\
33, \mathrm{CC} \\
34-1,98-99 \\
34-2,78-79 \\
\end{array}$ & I & & & & $\begin{array}{ll}R & F \\
& R \\
& F\end{array}$ & & & $\begin{array}{l}\mathrm{R} \\
\mathrm{R} \\
\mathrm{R}\end{array}$ & $\begin{array}{l}\mathrm{R} \\
\mathrm{R} \\
\mathrm{F} \\
\mathrm{R} \\
\mathrm{R}\end{array}$ & \\
\hline $\begin{array}{l}34, \mathrm{CC} \\
35-2,77-78\end{array}$ & & & & & $\begin{array}{l}\mathrm{F} \\
\mathrm{F}\end{array}$ & & & R & $\mathrm{R}$ & \\
\hline
\end{tabular}




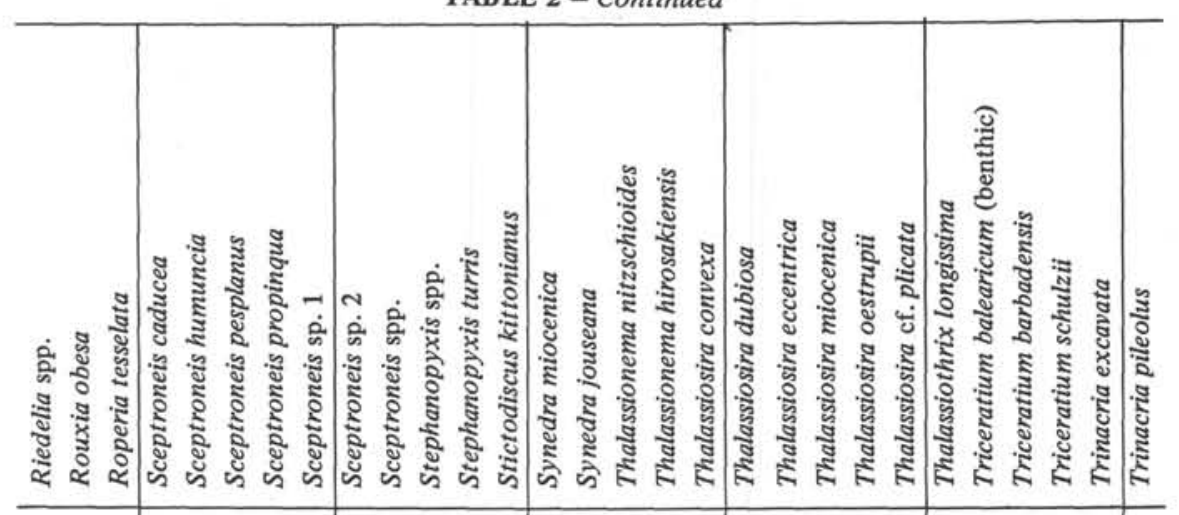

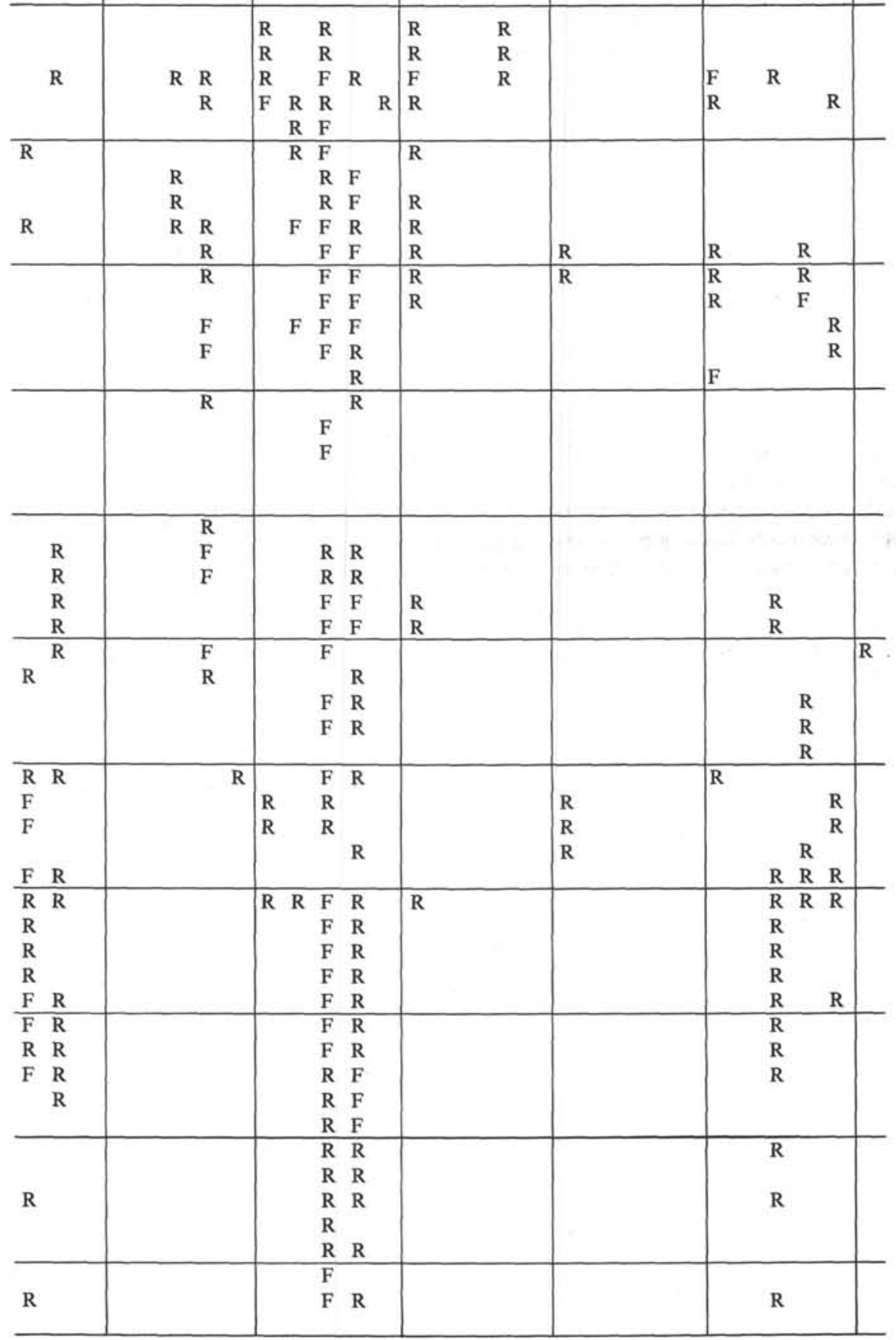




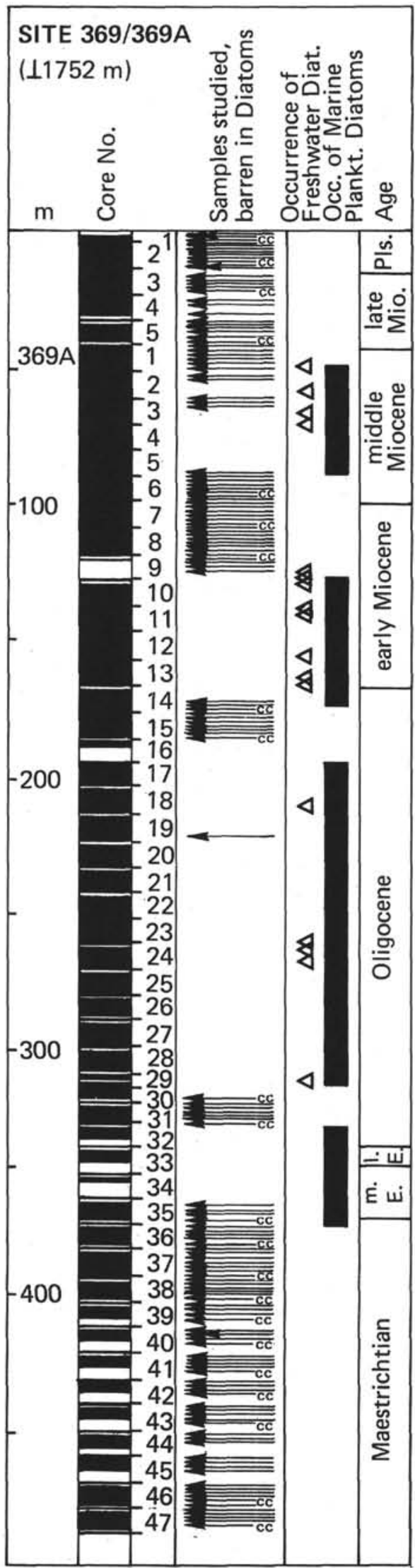

Figure 4. Site 369 and Hole $369 \mathrm{~A}$ summary figure. Arrows indicate samples barren in diatoms, triangles occurrence of displaced fresh-water diatoms. Age assignments are taken from the site reports (this volume).

\section{Site 369 (Holes 369 and 369A)}

This section consists predominantly of nannofossil marls with siliceous components in the Eocene through Miocene intervals (Figure 4). Samples barren in opal phytoplankton are listed as arrows in Figure 4.

Ranges of selected diatom species are compiled in Figures 5 and 6. The range of Annellus californicus Core 12 through the base of Core 9 places the middle-early Miocene boundary in between Cores 10 and 11 (for definition see Schrader and Burckle, in press).

Displaced fresh-water diatoms, phytoliths, and displaced marine benthic diatoms are listed in Figure 4.

\section{Site 370}

None of the 169 samples from Cores 1-51 (except Cores 4 and 11) in the deep basin off Morocco contain opal phytoplankton remains.

\section{ACKNOWLEDGMENT}

I thank the scientific party of Leg 41 for providing diatom shore-based samples. This paper was supported by the Deutsche Forschungsgemeinschaft and by the National Science Foundation of America.

\section{REFERENCES}

Burckle, L.H., 1972. Late Cenozoic planktonic diatom zones from the eastern equatorial Pacific: Nova Hedwig., Beihft., v. 39, p. 217-246.

Hustedt, F., 1930-1959. Die Kieselalgen Deutschlands, Osterreichs und der Schweiz. In Rabenhorst, L. (Ed.), Kryptogamen-Flora von Deutschland, Osterreich und der Schweiz, v. 7: Leipzig.

Kolbe, R.W., 1954. Diatoms from equatorial Pacific cores: Rept. Swedish Deep-Sea Exped., v. 6, p. 49.

Schrader, H.-J., 1974. Cenozoic marine planktonic diatom stratigraphy of the tropical Indian Ocean. In Fisher, R.L., Bunce, E.T. et al., Initial Reports of the Deep Sea Drilling Project, Volume 24: Washington (U.S. Government Printing Office), p. 887-967.

Schrader, H.-J. and Burckle, L.H., in press. Marine diatom biostratigraphy, its correlation to other microfossil zonations and to the paleomagnetic record. In Riedel, W.R. and Saito, T. (Eds.), Marine plankton and sediments, Symposium volume.

Schrader, H.-J. and Fenner, J., 1976. Norwegian Sea Cenozoic diatom biostratigraphy. In Talwani, M., Udintsev, G. et al., Initial Reports of the Deep Sea Drilling Project, Volume 38: Washington (U.S. Government Printing Office), p. 921-1099.

Simonsen, R., 1974. The diatom plankton of the Indian Ocean expedition of R/V "Meteor" 1964-1965. "Meteor" Forschung.-Ergebn., D 19, p. 66. 


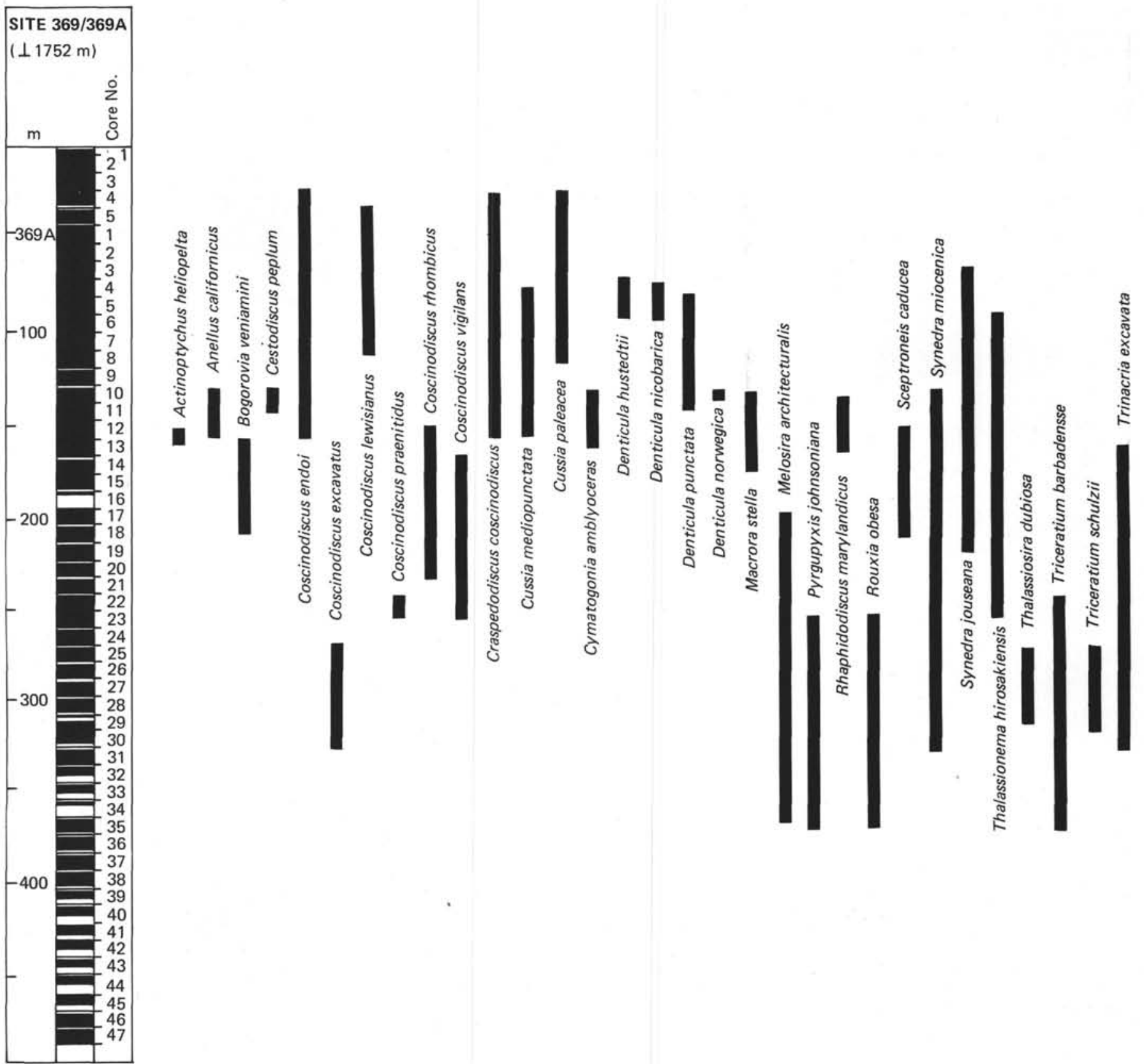

Figure 5. Occurrence of selected diatoms at Site 369 and Hole $369 \mathrm{~A}$ (range chart). 


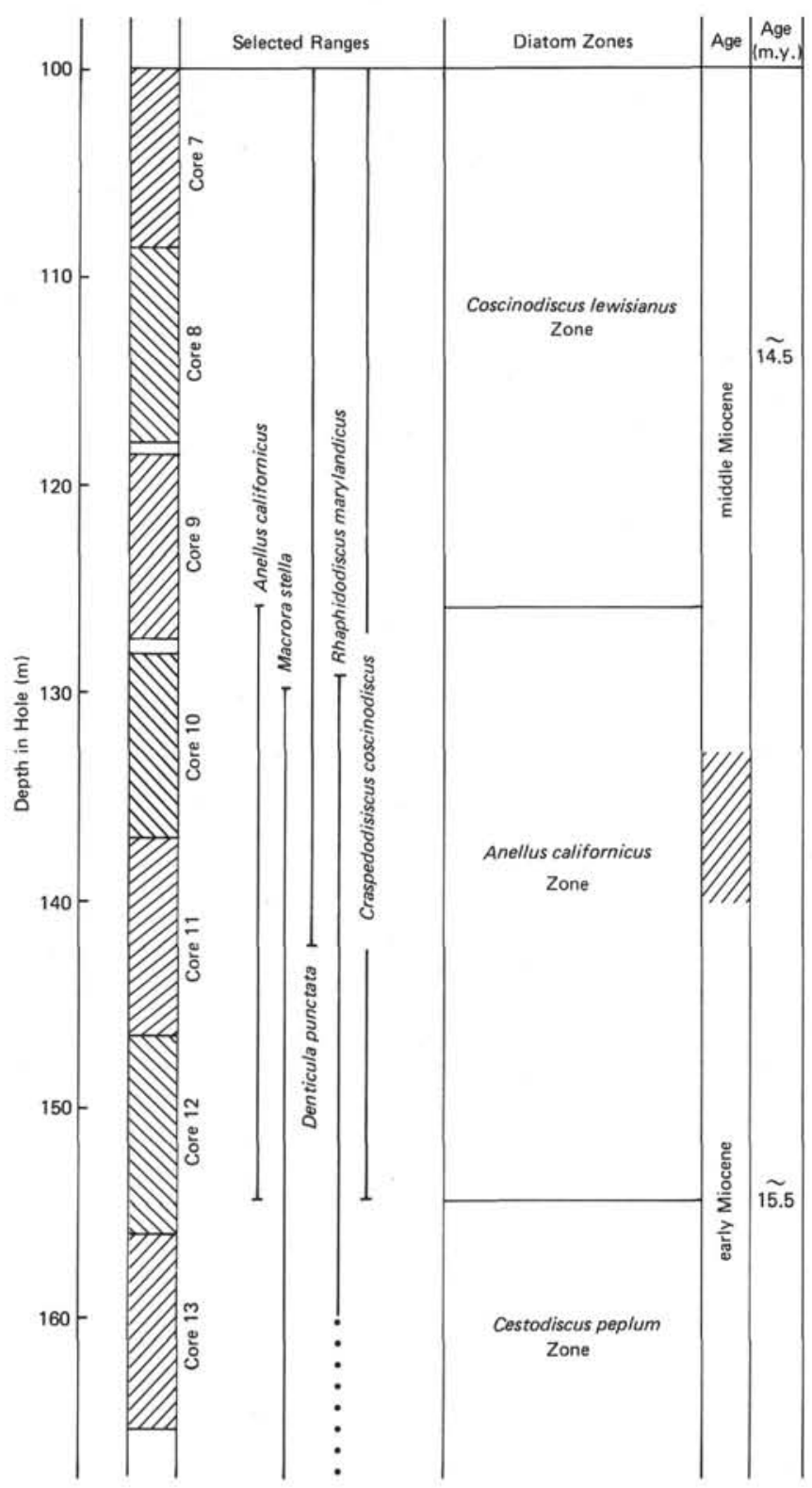

Figure 6. Range chart of important early to middle Miocene diatoms at Hole 369A. Diatom zones are those of Schrader and Burkle (in press). The early-middle Miocene boundary is placed between Cores 10 and 11 . 